\title{
A REVIEW ON INVOLVEMENT OF TREGS IN HUMAN SQUAMOUS CELL CARCIOMA BASED UPON DIFFERENTIAL EXPRESSION ON TUMOUR INFILTRATION
}

\author{
G.Rajeev Manideep \\ Dept. of Bio Medical Engineering \\ Saveetha School of Engineering, \\ Saveetha University
}

\begin{abstract}
Cancer development is extremely associated to the physiological condition of the tumour microenvironment (TME). Despite the present heterogeneousness of tumours from an equivalent or from completely different anatomical locations, common options will be found within the TME maturation of epithelialderived tumours. Genetic alterations in tumour cells lead to dysplasia, uncontrolled growth, resistance to cell death, and metabolic shift towards anaerobic metabolic process (Warburg effect). These events produce drive, aerophilic stress associate degreed pathology at intervals the TME triggering an adjustment of the animate thing matrix (ECM), a response from neighbour stromal cells (e.g., fibroblasts) and immune cells (lymphocytes and macrophages), causation maturation and, ultimately, leading to metastasis. Exosomes secreted by TME cells square measure central players all told these events. The TME profile is dominant on prognosis and impacts effectiveness of anti-cancer therapies. Hence, an enormous effort has been created to develop new therapeutic methods towards a a lot of economical targeting of TME. These efforts focus on: (i) therapeutic methods targeting TME elements, extending from standard medicine, to combined therapies and nanomedicines; and (ii) the event of models that accurately match the TME for bench investigations, as well as tumour-tissue explants, "tumour on a chip" or cellular tumour-spheroids [1].
\end{abstract}

Keywords: Tumour microenvironment, tumour development, cancer therapy, models for tumour microenvironment study, nanomedicines

\section{INTRODUCTION}

The development of effective anti-cancer therapies has been challenged by the quality of tumours. The growth heterogeneousness is exacerbated throughout the progression of the cancer at the side of the maturation of the cellular and single-celled elements of the growth niche-the growth

\author{
D. Jenila Rani \\ Dept. of Bio Medical Engineering \\ Saveetha School of Engineering \\ Saveetha University
}

microenvironment (TME). The TME consists of animate thing matrix (ECM), stromal cells (such as fibroblasts, mesenchymal stromal cells, pericytes, sometimes adipocytes, blood and body fluid vascular networks) and immune cells (including $\mathrm{T}$ and $\mathrm{B}$ lymphocytes, natural killer cells and Tumour-associated macrophages) [2]. The TME has more and more been shown to dictate aberrant tissue perform and to play an essential role within the future evolution of malignancies. animal tissue tumours show common options that allow the setting of hallmarks that outline cancer progression. growth initiation relies on a fancy series of biological events occurring on a standard cell that may lead to dysplasia, uncontrolled growth and resistance to death. As growth cells continue proliferation, the growth will increase in size with AN associated transforming of the TME. this can be induced by drive, anemophilous stress and pathology, thanks to AN alteration of growth cells metabolism, leading to abnormally, that is that the look of a heterogeneous population of tumoural cells with completely different genetic and phenotypical traits. These events area unit musical organisation by autocrine and paracrine communications with stromal cell and system adjacent to the growth, coupled to AN raised ECF pressure. Once again, autocrine and paracrine communications between TME cells induce TME maturation and growth progression, leading to raised stiffness of the animate thing matrix, formation of blood and liquid body substance vessels, attainable look of death regions and metastasis. Figure one may be a schematic illustration of the main events of growth progression [3].

Tumour development is very addicted to the precise TME, that is paramount on prognosis and impacts therapy potency. Understanding however the composition of the TME changes throughout tumour development might leave the event of therapeutic methods ready to tackle the tumour at a particular organic process stage. The study of TME throughout tumour development reveals prognostic biomarkers that will be used for imaging or for liquid diagnostic assay analysis, each vital to pick the foremost appropriate medical aid. This review 


\section{International Journal of Engineering Applied Sciences and Technology, 2020 Vol. 4, Issue 12, ISSN No. 2455-2143, Pages 570-575 \\ Published Online April 2020 in IJEAST (http://www.ijeast.com)}

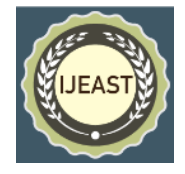

summarizes this information on the key players/events concerned in TME maturation of the first tumour which will induce or disrupt cancer progression. in addition, we tend to discuss therapeutic methods targeting these events, from customary current medicine to nanomedicine-based approaches and future strategies to watch the TME in vitro [4].

\section{ORIGINS OF TUMOUR-INFILTRATING TREG CELLS}

Treg cells, similarly as alternative subsets of $\mathrm{T}$ cells, will develop in thymus by the stimulation of self-antigens. These Tregs square measure termed as thymic Treg cells or natural Treg cells (tTregs or nTregs). The important role of thymic Treg cells in maintaining medicine tolerance has been incontestable by the observation that thymectomy of 3-day previous baby mice induced T-cell-mediated pathology. On the opposite hand, Tregs cells is differentiated from naïve $\mathrm{T}$ cells upon matter stimulation beneath sure protein conditions in peripheral tissues. These Treg cells square measure termed as peripheral Treg cells (pTregs) or induced Treg cells (iTregs). Peripheral Treg cells phenotypically correspond thymic Tregs, and each of them have similar constitution characteristics and comparable restrictive operate in suppressing immune responses. Maintaining of Treg restrictive operate needs the expression of transcription issue Foxp3, whose operate itself is regulated by a spread of posttranslational modifications, and Treg lineage stability is additionally determined by the institution of Treg cell-specific CpG hypomethylation pattern induced by lymphocyte receptor stimulation. In the context of neoplasm, there square measure many attainable origins accounting for the accrued Treg cell numbers in tumour [5].

\subsection{Treg cells are selectively recruited to tumour sites by a variety of chemokines:}

Hypoxia could be a common feature of neoplasm microenvironment, that drives growing and promotes neoplasm progression. drive induced the expression of chemotactic factors CCL28 in sex gland cancer and cancer of the liver, that promoted the accomplishment of CCR10+ Treg cells and also the institution of immune restrictive surroundings. Moreover, drive increased the essential secretion of VEGFA by tumour-infiltrating Treg cells, that promoted a pro-angiogenic neoplasm surroundings also. newer studies advised that FOXP3-expressing exocrine gland ductal carcinoma (PDAC) recruited Treg cells by secretion of CCL5, and selective depletion of Treg cells by CCL5 blockade could facilitate the antineoplastic response in PDAC patients [6]. CCL8/CCR5 sign axis drove Treg accomplishment to the lungs of mice bearing pathological process primary neoplasms and influenced the event of tumour metastases. Treg cells may even be recruited to the neoplasm during a CCR5-dependent manner and contributed to skin epithelial cell cancer (SSC) and body part cancer (CRC) development. The CCR4/CCL22 axis was highlighted for Treg accumulation in mouse malignant melanoma metastasis model. curiously, body covering overexpression of $\mathrm{Ccl} 22$ by factor gun vaccination may divert Treg cells removed from neoplasm and ameliorate neoplasm metastasis, so inhibiting reaction facet effects caused by immune stop medical specialty. There are a unit some tumour-associated cells concerned within the accomplishment of Treg cells through the oversized quantity of CCL20 to counterpoint CCR6+ regulative $\mathrm{T}$ cells and promoted the event of body part cancer. Similarly, macrophages and glia at intervals the brain tumour microenvironment made CCL2 that was vital for recruiting restrictive CCR4+ Treg also as CCR2+Ly-6C+ monocytic MDSCs. Besides, the clinical and diagnosis information showed that Indoleamine two,3-dioxygenase (IDO) multiplied the accomplishment of Treg cells to market tumour outgrowth. Inversely, artificial language deficiency decreased Treg accomplishment and increased T-cell-mediated neoplasm rejection [7].

\subsection{Conventional CD4+ T cells are converted to Treg cells in tumour:}

It was reported that IDO-expressing acute chronic granulocytic leukaemia (AML) cells could directly convert CD4 T cells into CD4+CD25+ Treg cells expressing surface CTLA-4 and FOXP3 mRNA. In fact, CTLA-4 signal is crucial for Foxp3+ Treg development. The treatment of IDO inhibitor 1-methyl tryptophan (1-MT) abrogated this effect and impaired T-cell tolerance in A20-bearing mice. In nonHodgkin lymphoma (NHL), NHL cells themselves were responsible for the increased numbers of Treg cells, and can induce Treg cells from CD25- PBMCs in vitro. Similarly, in follicular lymphoma (FL), malignant B cells alone, without artificial TCR stimulation, could induce conventional $\mathrm{T}$ cells to express Foxp3 with regulatory function via a cell-cell contact fashion. Moreover, T cells isolated from FL or normal peripheral blood were equally susceptible to being converted by tumour B cells, indicating that this effect was independent of the T cell background. Malignant B cells were also reported to induce the conversion of CD4 T cells to Treg cells through PD-1/B7-H1 pathway in B-Cell Non-Hodgkin Lymphoma. Exposure to immature dendritic cells (DCs) which loaded with apoptotic Cutaneous T-cell lymphoma (CTCL) cells conferred CD4+ CTCL cells acquiring regulatory T cell phenotypes, like expression of CD25, CTLA-4, Foxp3 and secretion of IL-10 and TGF- $\beta$. Inversely, blocking the expression or transport of DC MHC Class 2 inhibited CTCL cell from adopting Treg features[8].

However, whether this effect is restricted for blood cancer or general among other kinds of cancers remains elusive. A recent study found that suppressive IL-17A+Foxp3+ and ex- 


\section{International Journal of Engineering Applied Sciences and Technology, 2020 Vol. 4, Issue 12, ISSN No. 2455-2143, Pages 570-575 \\ Published Online April 2020 in IJEAST (http://www.ijeast.com)}

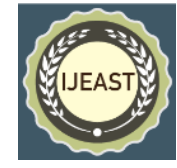

Th17 IL-17AnegFoxp3+ Treg cells are a source of tumourassociated Treg cells which were converted from IL$17 \mathrm{~A}+$ Foxp3neg cells in ovarian or colorectal cancer-bearing mice. These suppressive cells exerted active aerobic glycolysis, which is metabolically characteristic of Th cells. On the other hand, studies with transgenic and knockout animals demonstrated that some genes play cell-intrinsic roles in determining Treg cell-lineage stability. Therefore, deficiency of these genes can convert Treg cells into Teff cells inversely with enhanced anti-tumour immunity. as an example, Helios deficiency within CD4 Treg cells led to instability of intratumourally but not systemic Tregs, and conversion of these Tregs into Taff cells within the transplantable melanoma (B16/F10) or colon adenocarcinoma (MC38). Similarly, Treg specific deletion of Nrp1 gene drove Treg cell fragility with increasing production of proinflammatory cytokine IFN- $\gamma$ in tumour without impacting Treg cell function elsewhere within the body, indicating Nrp1 signalling could be specifically targeted to modulate intratumourally Treg cell activity and enhance anti-tumour immune response. Moesin, a member of the ezrin-radixinmoesin (ERM) family of proteins, plays a critical role in augmenting optimal TGF- $\beta$ signaling and facilitates efficient development of iTreg cell. Moesin-deficient mice were protected against recurrence of B16 melanoma tumour after adoptive $\mathrm{T}$ cell transfer because of the impaired conversion to FOXP3+ iTregs and compromised suppressive capacity. Although these studies suggest the potential perturbation of Treg cell stability and conversion of Treg cells in animal tumour models through targeting a specific specific signaling pathway, the thanks to manipulate Treg stability and elegance related therapeutic intervention for human cancer therapy remain to be further explored [9].

\subsection{Tumour microenvironment promotes Treg cells proliferation and stability:}

Tumour microenvironment contains a spread of things that facilitate Treg cell proliferation. The increased Treg cell numbers in tumour are attributed to the cell activation and expansion, which is supported by the analysis of the TCR repertoires from tumour-infiltrating effector and regulatory $\mathrm{T}$ cells. TC-1 tumour-infiltrating Teff and Treg cells displayed biased TCR repertoire respectively and characteristic of antigen-driven clonal expansions. Similarly, Treg cells were significantly enriched within the chemical carcinogen 3-methylcholanthrene (MCA)induced tumours (fibrosarcoma's). TCR repertoires of antigen-experienced tumour-infiltrating Tconvs and Tregs were largely distinct and non-overlapping, implying that tumour microenvironment promotes Treg cell proliferation or survival rather than conversion from Tongs cells in tumours. additionally, activated Treg cells (defined as CD4+CD45RA-Foxp3high) could be preferentially expanded in carcinoma (CC) and non-small cell carcinoma (NSCLC). However, these Treg cells were phenotypically plastic with the expression of Rots transcription factor and production of pro-inflammatory cytokine IL-17[10].

\subsection{Features of tumour-infiltrating Treg cells:}

CpG hypomethylation of the Foxp3 Treg-specific demethylated region (TSDR) could also be an indicator of stable nTregs, which distinguishes nTreg cells from other cell types including TGF $\beta$-inducing iTreg cells and activated FOXP3-expressing effector T cells. The tumourinfiltrating Tregs from a spread of mouse tumours and human tumours (NSCLC and ovarian) exhibited a uniform pattern of Foxp3 TSDR hypomethylation. Moreover, TGF$\beta$ neutralization by TGF- $\beta$-Trap didn't impact intratumorally Treg frequency and accumulation, indicating that nTreg-like cells rather than iTregs predominantly populate tumours in mice and humans. By using methylation-specific quantitative polymerase chain reaction (MS-qPCR) assay, a uniform study has demonstrated that a majority of these suppressive Treg cells are functional nTreg cells within the human colorectal cancer. These observations are in line with the hypothesis of recruitment rather than conversion about the origins of Treg cells in tumour[11].

As mentioned above, given Treg cells are highly activated and proliferative in animal cancer models or cancer patients, tumour-infiltrating Treg cells require metabolic reprogramming to support their function and expansion. Tumour microenvironment is metabolically abnormal because of the poor replenishment of nutrients like glucose, glutamine and tryptophan while being enriched with acid and kynurenines. Alessia Angelin et al. reported that Treg cells adopted a selective metabolic advantage in low-glucose, high-lactate environments which is characteristic of the tissues with ischemic injury or the microenvironment surrounding solid tumours. Foxp3 expression induced in iTreg cells or retrovirally transduced generally $\mathrm{T}$ cells increased oxygen consumption rates (OCR) and biological process (OXPHOS) for his or her energy production, while inhibited Myc expression and glycolysis in these cells. However, Treg division and suppressive function were unaffected by the exposure to L-lactate which was enriched within the tumour microenvironment. Of note, Treg metabolic advantage didn't depend upon the facility to use L-lactate as an alternate fuel source when glucose was sparse, but rather, the resistance to the depletion of intracellular NAD pool resulting from the oxidation of Llactate to pyruvate by lactate dehydrogenase (LDH), which significantly impairing Teff function and proliferation on the contrary[12]. 
International Journal of Engineering Applied Sciences and Technology, 2020

Vol. 4, Issue 12, ISSN No. 2455-2143, Pages 570-575

Published Online April 2020 in IJEAST (http://www.ijeast.com)

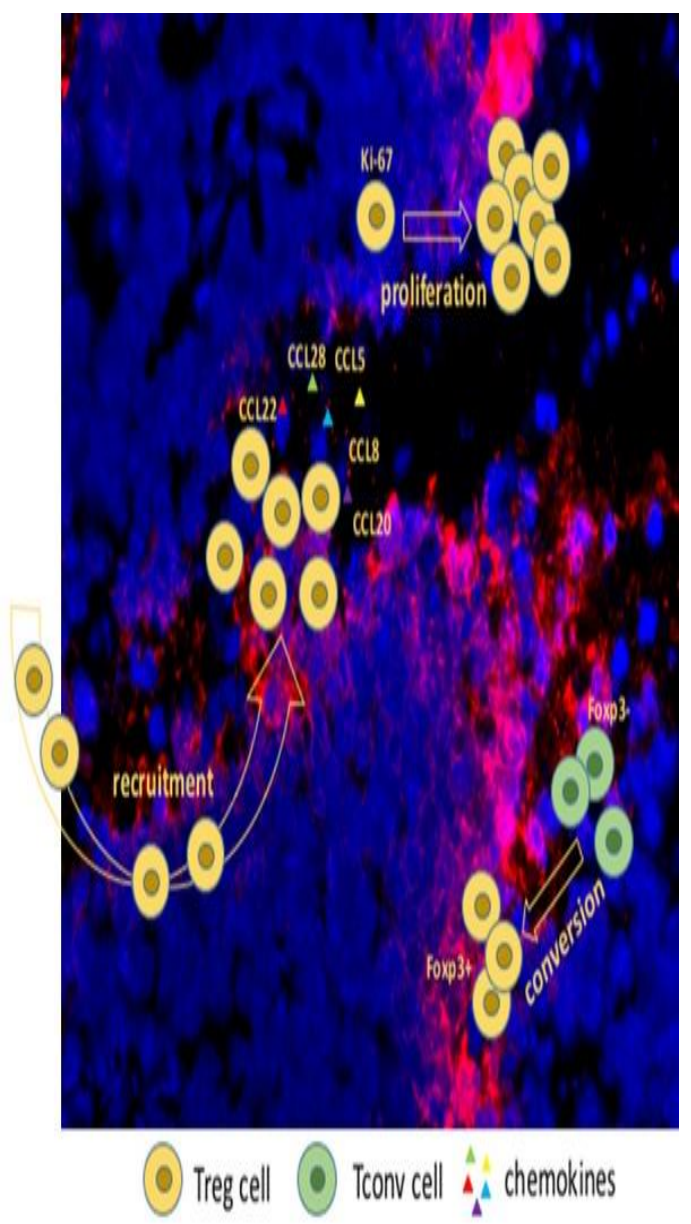

[SOURCE: TUMOUR-INFILTRATING REGULATORY T CELLS: ORIGINS AND FEATURES, GUOPING DENG].

\subsection{TARgeting Hypoxia ANd ACIDOSIS:}

The partial oxygen pressure within the TME is usually but in normal tissues. additionally, the TME may experience two sorts of low oxygenation events, chronic and acute/cycling hypoxia[13].

HIF-1 could also be a dimeric protein complex composed by two subunits that are constitutively expressed within the cells, the cytoplasmic-oxygen dependent subunit alpha, HIF- $1 \alpha$, and thus the nuclear subunit beta, HIF-1 $\beta$. Under hypoxia, the lower activity of PHD-2 allows the translocation of HIF- $1 \alpha$ to the nucleus, interacting with the HIF-1 $\beta$ subunit and, in conjunction with the transcriptional co-activators $\mathrm{P} 300$ and CREB binding protein (CBP), binds to the hypoxia-responsive elements located at the promoter regions of over 100 genes involved in hypoxia response. This response to low oxygen pressure levels includes regulation of genes involved in glucose metabolism, cell proliferation, angiogenesis, macrophage polarization into tumour-associated macrophages (TAM) and metastasis[14].

Among these, Topotecan could also be a topoisomerase 1 inhibitor, FDA approved and used as second line treatment of ovarian and tiny cell carcinoma. Topotecan has been studied during a clinical trial for treatment of refractory advanced solid neoplasms expressing HIF-1 $\alpha$. additionally, other clinical trials include: evaluation of Metformin effect in tissue oxygenation of head and neck somatic cell carcinoma; the phase 4 trial study of the effect of Enviroximes (RAD001) in patients with advanced renal cell carcinoma; the phase 2 study of the effect of Enviroximes (RAD001) conjugated with Levantine in renal cell carcinoma; and thus the phase 2 study of pharmacodynamics of Digoxin (DIG-HIF1) in newly diagnosed operable carcinoma .Several compounds and therapies were designed to tackle HIF-1 or its targets to avoid tumour progression. Among these, Topotecan could also be a topoisomerase 1 inhibitor, FDA approved and used as second line treatment of ovarian and tiny cell carcinoma [15]. Topotecan has been studied during a clinical trial for treatment of refractory advanced solid neoplasms expressing HIF-1 $\alpha$. additionally, other clinical trials include: evaluation of Metformin effect in tissue oxygenation of head and neck somatic cell carcinoma; the phase 4 trial study of the effect of Everolimus (RAD001) in patients with advanced renal cell carcinoma; the phase 2 study of the effect of Everolimus (RAD001) conjugated with Lenvatinib in renal cell carcinoma; and thus the phase 2 study of pharmacodynamics of Digoxin (DIG-HIF1) in newly diagnosed operable carcinoma[16].

\section{CONCLUSION}

It is now well-documented that Treg cells contribute to the establishment of immune suppressive tumour microenvironment and promote the expansion of various kinds of tumours. The origins of Treg cells in tumour are diverse according to different cancer types. Tumourinfiltrating Treg cells are highly activated and proliferative. Treg cells have metabolic advantages to adopt to the tumour microenvironment. Understanding the origins and phenotypic features of tumour-infiltrating Treg cells may, therefore, help us to style therapeutic strategies targeting Treg cells to strengthen anti-tumour immune responses together of other immunotherapies. to this end, there are two interesting questions remained to be answered [17]. How do Treg cells sustain their lineage stability under extremely stressful conditions. Actually, Treg cells may become unstable and plastic under certain conditions like hypoxia. Hypoxia induced hypoxia- 


\section{International Journal of Engineering Applied Sciences and Technology, 2020 Vol. 4, Issue 12, ISSN No. 2455-2143, Pages 570-575 \\ Published Online April 2020 in IJEAST (http://www.ijeast.com)}

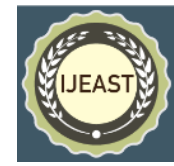

inducible factor 1 (HIF-1), a key metabolic sensor, which enhanced Th17 differentiation but inhibited Treg differentiation during $\mathrm{T}$ cell lineage commitment. What are the potential interplays between Treg cells with other type cells like malignant tumour cells, stroma cells and immune cells. Tumour-infiltrating immune cells are heterogeneous among tumour types, and vary from patient to patient. Tumour-infiltrating Treg cells can promote immune tolerance by suppressing tumour-associated DC immunogenicity in pancreatic ductal adenocarcinoma (PDA). Answers to those questions would help us better understand the biology of tumour-infiltrating Treg cells and elegance rational clinic interventions for tumour therapy[18].

\section{REFERENCES}

1. Nishizuka Y, Sakakura T. Thymus and reproduction: sex-linked dysgenesia of the gonad after neonatal thymectomy in mice. Science. 1969; 166:753-755.

2. Chen W, Jin W, Hardegen N, Lei KJ, Li L, Marinos N, McGrady G, Wahl SM. Conversion of peripheral $\mathrm{CD} 4$ naive $\mathrm{T}$ cells to $\mathrm{CD} 4+$ CD25+ regulatory $\mathrm{T}$ cells by TGF-beta induction of transcription factor Foxp3. J Exp Med. 2003; 198:1875-1886.

3. Zheng SG, Gray JD, Ohtsuka K, Yamagiwa S, Horwitz DA. Generation ex vivo of TGF-betaproducing regulatory $\mathrm{T}$ cells from $\mathrm{CD} 4+$ CD25- precursors. JImmunol.2002;169:41834189.

4. Lin X, Chen M, Liu Y, Guo Z, He X, Brand D, Zheng SG. Advances in distinguishing natural from induced Foxp3(+) regulatory T cells. Int J Clin Exp.Pathol.2013;6:116-123.

5. Hori S, Nomura T, Sakaguchi S. Control of regulatory $\mathrm{T}$ cell development by the transcription factor Foxp3. Science. 2003; 299:1057-1061

6. Deng G, Xiao Y, Zhou Z, Nagai Y, Zhang H, Li B, Greene MI. Molecular and biological role of the FOXP3 N-terminal domain in immune regulation by $\mathrm{T}$ regulatory/suppressor cells. Exp Mol Pathol. 2012; 93:334-338.

7. Deng G, Nagai Y, Xiao Y, Li Z, Dai S, Ohtani T, Banham A, Li B, Wu SL, Hancock W, Samanta A, Zhang H, Greene MI. Pim-2 kinase influences regulatory $\mathrm{T}$ cell function and stability by mediating Foxp3 protein Nterminal phosphorylation. J Biol Chem. 2015; 290:20211-20220.

8. Xiao Y, Nagai Y, Deng G, Ohtani T, Zhu Z, Zhou Z, Zhang H, Ji MQ, Lough JW, Samanta A, Hancock WW, Greene MI. Dynamic interactions between TIP60 and p300 regulate FOXP3 function through a structural switch defined by one lysine on TIP60. Cell Rep. 2014; 7:1471-1480.

9. Ohkura N, Hamaguchi M, Morikawa H, Sugimura K, Tanaka A, Ito Y, Osaki M, Tanaka Y, Yamashita R, Nakano N, Huehn J, Fehling HJ, Sparwasser T, Nakai K, Sakaguchi $\mathrm{S}$. T cell receptor stimulation-induced epigenetic changes and Foxp3 expression are independent and complementary events required for Treg cell development. Immunity.

10. Facciabene A, Peng X, Hagemann IS, Balint K, Barchetti A, Wang LP, Gimotty PA, Gilks CB, Lal P, Zhang L, Coukos G. Tumour hypoxia promotes tolerance and angiogenesis via CCL28 and T(reg) cells. Nature. 2011; 475:226-230.

11. Wang X, Lang M, Zhao T, Feng X, Zheng C, Huang C, Hao J, Dong J, Luo L, Li X, Lan C, Yu W, Yu M, Yang S, Ren H. Cancer-FOXP3 directly activated CCL5 to recruit FOXP3(+)Treg cells in pancreatic ductal adenocarcinoma.Oncogene.2017;36:30483058 .

12. Halvorsen EC, Hamilton MJ, Young A, Wadsworth BJ, LePard NE, Lee HN, Firmino $\mathrm{N}$, Collier JL, Bennewith KL. Maraviroc decreases CCL8-mediated migration of CCR5(+) regulatory $\mathrm{T}$ cells and reduces malignant tumour growth within the lungs. Oncoimmunology. 2016;5: e1150398.

13. Ward ST, Li KK, Hepburn E, Weston CJ, Curbishley SM, Reynolds GM, Hejmadi RK, Bicknell R, Eksteen B, Ismail T, Rot A, Adams DH. the consequences of CCR5 inhibition on regulatory $\mathrm{T}$-cell recruitment to colorectal cancer. Br J Cancer. 2015; 112:319-328.

14. Klarquist J, Tobin K, Farhangi Oskuei P, Henning SW, Fernandez MF, Dellacecca ER, Navarro FC, Eby JM, Chatterjee S, Mehrotra S, Clark JI, Le Poole IC. Ccl22 diverts T regulatory cells and controls the expansion of melanoma. Cancer Res. 2016; 76:6230-6240.

15. Liu J, Zhang N, Li Q, Zhang W, Ke F, Leng Q, Wang H, Chen J, Wang H. Tumour-associated macrophages recruit CCR6+ regulatory $\mathrm{T}$ cells and promote the event of colorectal cancer via enhancing CCL20 production in mice.LoSOne.2011;6:e19495.

16. Chang AL, Miska J, Wainwright DA, Dey M, Rivetta CV, Yu D, Kanojia D, Pituch KC, Qiao J, Pytel P, Han Y, Wu M, Zhang L, Horbinski CM, Ahmed AU, Lesniak MS. CCL2 produced 
by the glioma microenvironment is important for the recruitment of regulatory $\mathrm{T}$ cells and myeloid-derived suppressor cells. Cancer Res. 2016; 76:5671-5682.

17. Wainwright DA, Balyasnikova IV, Chang AL, Ahmed AU, Moon KS, Auffinger B, Tobias AL, Han Y, Lesniak MS. IDO expression in brain tumours increases the recruitment of regulatory $\mathrm{T}$ cells and negatively impacts survival. Clin Cancer Res. 2012; 18:61106121.

18. Zheng SG, Wang JH, Stohl W, Kim KS, Gray JD, Horwitz DA. TGF-beta requires CTLA-4 early after $\mathrm{T}$ cell activation to induce FoxP3 and generate adaptive CD4+CD25+ regulatory cells. J Immunol. 2006; 176:332.

19. Curti A, Pandolfi S, Valzasina B, Aluigi M, Isidori A, Ferri E, Salvestrini V, Bonanno G, Rutella S, Durelli I, Horenstein AL, Fiore F, Massaia M, Colombo MP, Baccarani M, Lemoli RM. Modulation of tryptophan catabolism by human leukemic cells leads to the conversion of CD25- into CD25+ T regulatory cells. Blood. 2007; 109:2871-2877.

20. Mittal S, Marshall NA, Duncan L, Culligan DJ, Barker RN, Vickers MA. Local and systemic induction of CD4+CD25+ regulatory $\mathrm{T}$-cell population by non-Hodgkin lymphoma. Blood. 2008; 111:5359-5370. 